\title{
Can there be consensus on whether vasectomy is a prostate cancer risk factor?
}

\author{
M. Minhaj Siddiqui ${ }^{1} \cdot$ Edward L. Giovannucci ${ }^{2,3} \cdot$ Lorelei A. Mucci $^{3}$
}

Received: 30 March 2021 / Revised: 7 May 2021 / Accepted: 19 May 2021 / Published online: 9 June 2021

(c) The Author(s), under exclusive licence to Springer Nature Limited 2021

A 40-year-old man consults his urologist about having a vasectomy. He is aware of vasectomy's effectiveness in preventing pregnancy, and he and his partner believe it is the best birth control option for their family. While the American Urological Association guidelines state that clinicians need not discuss prostate cancer in pre-vasectomy counseling of patients, many men are aware of published reports on vasectomy and prostate cancer. If the patient is a Black man or has a prostate cancer family history, he may already be concerned about his higher prostate cancer incidence and mortality $[1,2]$. The patient may thoughtfully ask how to weigh potential risks and benefits. The urologist's response will depend on her understanding of the state of evidence of vasectomy and prostate cancer, of the complex methodologic issues, and for her patient, whether his baseline risk factors will influence future cancer occurrence. The above clinical scenario is not straightforward. A rancorous debate has ensued over three decades, since an early report of a positive association between vasectomy and prostate cancer [3]. Several epidemiologic investigations have been published since, some methodologically rigorous, others less so, and the research and clinical communities seem no closer to consensus.

A meta-analysis of 18 cohort and 36 case-control studies appears in this issue of Prostate Cancer and Prostatic Diseases [4]. The authors, appreciating the etiologic and biologic heterogeneity of prostate cancer [5], examined overall prostate cancer and clinical subtypes based on stage, grade, and

Lorelei A. Mucci

lmucci@hsph.harvard.edu

1 Department of Surgery, University of Maryland School of Medicine, Baltimore, MD, USA

2 Departments of Nutrition, Harvard T.H. Chan School of Public Health, Boston, MA, USA

3 Epidemiology, Harvard T.H. Chan School of Public Health, Boston, MA, USA mortality. Prostate specific antigen (PSA) screening has changed the interpretation of risk factor associations with overall prostate cancer, as most cancers diagnosed in epidemiologic studies in the PSA-era are low-risk.

To explore potential issues, the authors considered differences in findings by level of bias (Newcastle-Ottawa Scale), duration of follow-up, and publication year. They report on associations with overall prostate cancer in studies accounting for PSA screening, although eFigure 5's title is unclear. PSA screening can be a powerful confounder, as men who undergo vasectomy often have closer medical follow-up and screening. The direction of bias depends on the outcome. On the one hand, PSA screening is strongly and positively associated with an overall prostate cancer diagnosis, inflating the relative risk estimate (Fig. 1A). On the other, screening reduces advanced and fatal prostate cancer. As such, confounding due to screening would bias the relative risk towards the null value (Fig. 1B).

What are the key findings from this meta-analysis? Across all 56 studies, vasectomy was associated with a small but statistically significant risk of overall prostate cancer (RR 1.18, 1.07-1.31). The relative risk was attenuated, although still significant, when limited to low risk of bias studies, including all cohort studies (RR 1.09, 1.04-1.14). Restricting to studies that adjusted for screening in multivariable models, the relative risk was 1.06 (1.04-1.09). The relative risk was higher in studies assessed to be of higher bias $(1.31,1.02-1.70)$. The interpretation of overall prostate cancer as an endpoint varies across studies, as it depends on the relative proportion of clinically significant vs. indolent cancers in a population.

Given the powerful confounding effect of PSA screening, could residual confounding account for the modestly excess overall prostate cancer? While we do not know this from the meta-analysis, the answer is potentially yes. In our Health Professionals Follow-up Study (HPFS) [6], tightly controlling for confounding by restricting to a highly screened subcohort attenuated the relative risk and 95\% confidence interval (RR $1.05,0.94-1.17)$ compared to a simpler adjustment (RR 1.10, 


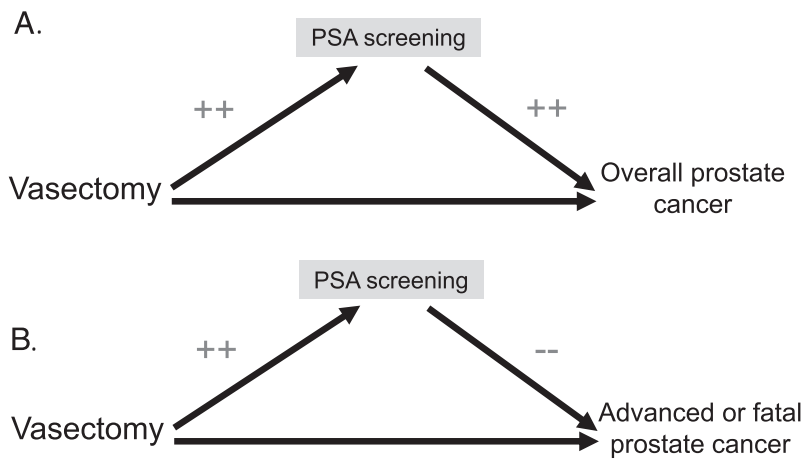

Fig. 1 Is a directed acyclic graph (DAG) representing the effect of PSA screening in prostate cancer epidemiology studies. A has overall prostate cancer as the endpoint, and $\mathbf{B}$ has advanced or fatal prostate cancer. In each figure, there is a directed arrow from vasectomy and PSA screening, as men who undergo vasectomy will often have closer medical evaluation and as a corollary greater frequency and intensity of PSA screening. A. PSA screening is strongly and positively associated with an overall prostate cancer diagnosis. Together with the positive association between vasectomy and PSA screening, this results in biasing the relative risk estimate for vasectomy and prostate cancer overall away from the null value, or inflating the relative risk. B Over time, PSA screening leads to a lower incidence of advanced prostate cancer and ultimately reduced prostate cancer mortality. In contrast to the bias for overall prostate cancer, confounding due to PSA screening biases the relative risk toward the null value.

1.04-1.17). Within the PLCO screening trial, the relative risk for vasectomy and prostate cancer was $1.11(1.03-1.20)$ in the usual care arm compared to $1.03(0.95-1.11)$ in the screening arm, minimally affected by confounding [7]. To our knowledge, no other individual study in the meta-analysis investigated screening's impact to such depth. The meta-analysis concludes that "From the current evidence, patients should be fully informed of the risk of prostate cancer before vasectomy." Given the potential of residual confounding, and the modest clinical significance of the absolute risk translated from the relative risk, we suggest caution in translating these findings into clinical practice.

Findings for clinically significant disease suggested a small excess risk of advanced, high-grade cancers, but no association with cancer mortality. There was no separate presentation of results accounting for PSA screening. However, as noted in the Health HPFS [6], the relative risk for lethal prostate cancer was $1.19(1.00-1.43)$ in the full cohort and $1.56(1.03-2.36)$ in the highly screened subcohort, highlighting the potential for screening to bias risk factor associations with advanced cancer towards the null. Still, is this a causal finding? A preclinical study supports the positive association of vasectomy and clinically significant prostate cancer, where vasectomized mice had increased incidence of poorly differentiated, increased proliferation of prostate tumors compared to sham mice [8]. As a call to action, the epidemiologic and clinical communities should unite to pool prospective cohort data, and investigate associations of vasectomy and advanced/lethal cancer risk carefully accounting for PSA screening intensity. Such an approach is needed to provide the evidence needed to make definitive interpretations of the findings.

In returning to the clinical scenario above, what is known about vasectomy and prostate cancer among at-risk men? Relatively little. Two multiethnic case-control studies [9, 10] from the 1990s reported associations specifically among Black men for prostate cancer overall, without adjustment for diagnostic intensity, and with mixed results. Such limited data is emblematic of a greater unmet need, for well-done prospective studies of prostate cancer etiology among Black men as well as men at increased genetic susceptibility, populations that suffers the greatest burden of prostate cancer.

There are two related issues remaining: Can we determine if relative risks of the magnitude seen for vasectomy and prostate cancer are causal? If so, are they clinically important? The former can be answered with the pooled cohort analysis described above. The latter may depend on a man's lifetime prostate cancer risk. While a relative risk of 1.2 has minimal clinical impact for a man at low susceptibility, his lifetime risk may be considerably higher if his baseline risk is already elevated.

\section{Compliance with ethical standards}

Conflict of interest The authors declare no competing interests.

Publisher's note Springer Nature remains neutral with regard to jurisdictional claims in published maps and institutional affiliations.

\section{References}

1. Siegel RL, Miller KD, Fuchs HE, Jemal A. Cancer Statistics, 2021. CA Cancer J Clin. 2021;71:7-33.

2. Barber L, Gerke T, Markt SC, Peisch SF, Wilson KM, Ahearn T, et al. Family history of breast or prostate cancer and prostate cancer risk. Clin Cancer Res. 2018;24:5910-7.

3. Mettlin C, Natarajan N, Huben R. Vasectomy and prostate cancer risk. Am J Epidemiol. 1990;132:1056-61. discussion 1062-1055

4. Xu Y, Yang W, Zhang K, Ma K, Xie H, Zhou J et al. Association between vasectomy and risk of prostate cancer: A systematic review and meta-analysis. Prostate Cancer Prostatic Dis. 2021; In press.

5. Hurwitz LM, Agalliu I, Albanes D, Barry KH, Berndt SI, Cai Q et al. Recommended definitions of aggressive prostate cancer for etiologic epidemiologic research. J Natl Cancer Inst. 2021;113: 727-34.

6. Siddiqui MM, Wilson KM, Epstein MM, Rider JR, Martin NE, Stampfer MJ, et al. Vasectomy and risk of aggressive prostate cancer: a 24-year follow-up study. J Clin Oncol. 2014;32:3033-8.

7. Shoag J, Savenkov O, Christos PJ, Mittal S, Halpern JA, Askin G, et al. Vasectomy and risk of prostate cancer in a screening trial. Cancer Epidemiol Biomark Prev. 2017;26:1653-9.

8. Kawahara T, Teramoto Y, Li Y, Ishiguro H, Gordetsky J, Yang Z et al. Impact of vasectomy on the development and progression of prostate cancer: preclinical evidence. Cancers (Basel). 2020;12: 2295. 
9. Hayes RB, Pottern LM, Greenberg R, Schoenberg J, Swanson GM, Liff J, et al. Vasectomy and prostate cancer in US blacks and whites. Am J Epidemiol. 1993;137:263-9.
10. John EM, Whittemore AS, Wu AH, Kolonel LN, Hislop TG, Howe GR, et al. Vasectomy and prostate cancer: results from a multiethnic case-control study. J Natl Cancer Inst. 1995;87:662-9. 